\title{
CRITICAL FACTORS AFFECTING THE UTILIZATION OF CLOUD COMPUTING
}

\author{
Alberto Daniel Salinas Montemayor ${ }^{1}$, Jesús Fabián Lopez ${ }^{2}$, Jesús Cruz Álvarez ${ }^{3}$
}

\begin{abstract}
This research presets the critical factors that influence the use of cloud computing, in case studies of large and medium-sized enterprises in the metropolitan area of Monterrey. The critical factors are found, according to literature: dependency provider; ignorance of the location, where the information, service knowledge, knowledge of laws, service offered by suppliers, is stored; cost; and information security, describing the research and development, which are significant and demonstrative in its impact.

We used Cronbach's alpha to check the validity of the measurement instrument and used a linear regression method to measure the significance of the variables. We also relied on some measuring instruments, such as Beta, R-squared, Standard Deviation, Anova, Tolerance, and Collinearity index to propose a model.
\end{abstract}

UDC Classification: 004.9, DOI: http://dx.doi.org/10.12955/cbup.v2.481

Keywords: cloud computing, internet, information technology, cost, services, security

\section{Introduction}

Information technology (IT) is changing the traditional way of doing things. For instance, people who work in companies or as a professional in any field of IT must use the internet, credit cards, payroll system, among other functions. This is a reason why the role of IT in business processes, such as manufacturing and sales, has expanded greatly. The first generation of computers was intended to keep records and monitor the operating performance of the company, but the information was not timely, e.g. the analysis obtained on a given day actually described what had happened a week earlier. Current advancement makes it possible to capture and use information at the same time it is generated via online processes. This does not only change the way of doing the job and the workplace, but also has a major impact on the way in which firms compete in the industry (Alter, 1999).

In particular, IT means the tools and methods used to collect, store, manipulate, or distribute information. IT is generally associated with computers and related technologies for decision-making (Bologna \& Walsh, 1997).

Using IT effectively can gain competitive advantages, but it also requires successful methods to maintain such benefits constantly as well as to provide resources and alternative courses of action to suit spontaneous requirements, as these benefits are not always permanent. The information system has to be modified and updated regularly in order to maintain continuous competitive advantages. The creative use of information technology can provide managers with a new tool to differentiate human resources, products and services from its competitors (Alter, 1999). This type of competitive prominence may bring about another group of strategies, such as a flexible system or infrastructure to allow production of a wider range of products at a lower price and in less time than the competition.

The information technology represents an increasingly important tool in business; however, implementing an information system for a company does not guarantee its immediate results, or even long term.

In recent years, there has been a new development in the computer for a new computing model called "cloud computing," which is based primarily on the development and expansion of the internet. Basically, this type of computing is the proposed use of the advantages offered by the internet to access both physical resources (hardware) and software that do not necessarily have to be present on the same equipment used.

\footnotetext{
${ }^{1}$ Alberto Daniel Salinas Montemayor, Universidad Autónoma de Nuevo León, Mexico, asalinas771@gmail.com

2 Jesús Fabián Lopez, Universidad Autónoma de Nuevo León, Mexico, fabian.lopezpz@gmail.com

${ }^{3}$ Jesús Cruz Álvarez, Universidad Autónoma de Nuevo León, Mexico, jesusphd@prodigy.net.mx
} 
Cloud computing is, then, a virtualization of computing resources that tries to optimize its use and reduce infrastructure costs. Also, for some, it is a form of sustainable computing since this model is intended to reduce energy consumption of both private users and companies, by relocating their IT systems toward cloud applications.

\section{Problem statement}

According to a report by the International Audit and Control Association Information Systems (ISACA, 2011), in Northern Mexico, 26 \% of companies have already started using cloud computing as a factor in corporate business strategy.

According to this report, $13 \%$ of the companies using cloud computing do so for essential services and data storage. With the scalability and flexibility of own cloud platform, systems can be configured to be shared by hundreds of individuals and organizations, vertically or horizontally, in business processes and how they interact with customers and suppliers. This will result in organizations redefining their processes and infrastructure to operate in the cloud. Despite the growing adoption, the issue of information security has become the main concern for organizations using this model.

The calculation in the cloud enables organizations to save on the expenses of acquiring and managing infrastructure, as well as can reuse those resources to continuously expand the business.

Rawal (2012) describes some significant factors in the adoption of government and business of cloud computing, but focuses more on the human factor, the reluctance, or refusal to use the cloud, and the security of the stored data. Another study by Geczy (2011), focusing on the services that a provider could offer, also mentions that this service could become more dependent than the operation of a business to a supplier. There are also many studies on the subject of information security. Mather, Kumaraswamy, \& Latif (2009) referred to the absence of an international security framework that should be present to protect the information stored in the cloud. Moreover, an article by Julisch \& Hall (2010) highlights the importance that providers must adequately manage the access to the information.

There are also studies that show how to make attractive business services with cloud computing. Sheelvant (2009) indicates the cost and the significant savings that can be obtained from hiring a service in the cloud, and also points out that resources optimization, smaller footprint, downsizing, among other factors, could lead to hiring an outside service. Several studies emphasize the importance of variables such as: unit suppliers, ignorance of the location where the information is stored, service knowledge, legal knowledge, services offered by suppliers, cost reduction, and information security.

Since there is no research that jointly determines the correlation between the critical factors and the extent of the use of computational cloud, this study seeks to analyze jointly and systematically the impact of the factors included in the literature.

\section{Hypothesis}

The following critical factors are examined, for relationship with greater use of cloud computing (Y), as part of our research hypothesis:

- Dependency of Providers (X1)

- Data information (X2)

- Knowledge Service (X3)

- Legal Knowledge (X4)

- $\quad$ Services offered by suppliers (X5) 
- $\quad$ Cost (X6)

- Information Security (X7)

\section{Universe and Sample}

The case study is focused on large and medium-sized companies in the metropolitan area of Monterrey. The information from the Ministry of Economy provided us with 8,755 large and mediumsized enterprises; the sample size used in this analysis is calculated using a simple formula shown in Figure 1.

\begin{tabular}{|l|}
\hline Figure 1: The formula for sample size calculation \\
$\qquad \mathrm{n}=\frac{\mathrm{z}^{2} \mathrm{pq}}{\mathrm{B}^{2}} \quad \mathrm{n}^{\prime}=\frac{\mathrm{n}}{\left.1+\frac{\mathrm{n}}{\mathrm{N}}-1\right)}$ \\
\hline Source: Suarez (2011) \\
\hline
\end{tabular}

Using the formula provided in Figure 1, we can calculate the sample size for this analysis. The calculation is shown in Figure 2. The resulting sample size (n') is 561.79.

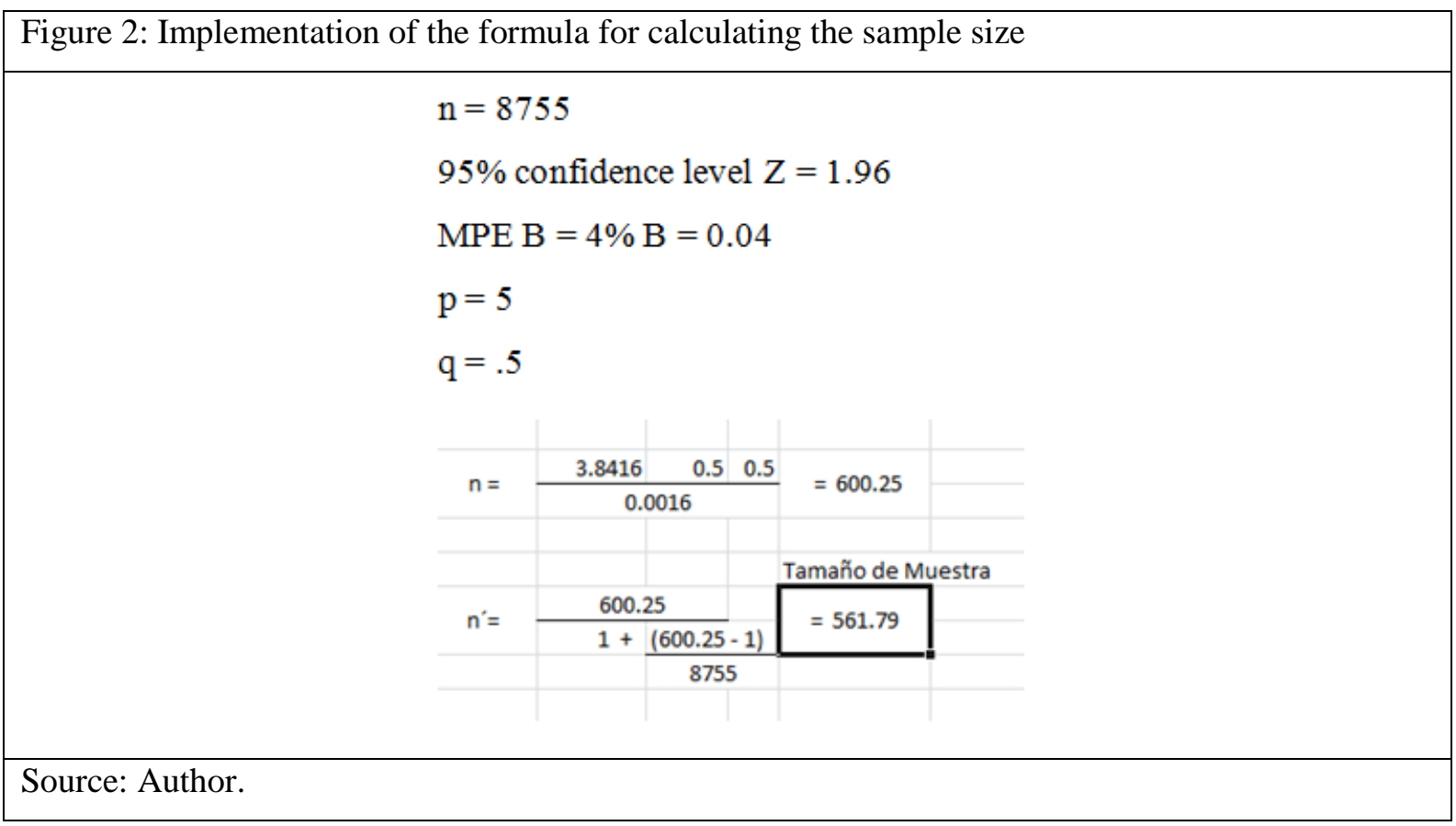

\section{Instrument}

The collection of information on the critical factors was conducted through a survey, which was designed with the intention of measuring the ratio of the dependent variables with respect to the extent of use of cloud computing.

\section{Type of test validity}

The calculation of the reliability of the instrument is the primary key to determining if the results extracted from it will be acceptable.

For the seven variables involved in the instrument, four of them returned significant value through a group of items. They are Dependency Provider (X1), Knowledge Service (X3), Costs (X6), and Information Security (X7). Their Cronbach’s Alpha values are shown in Table 1. 


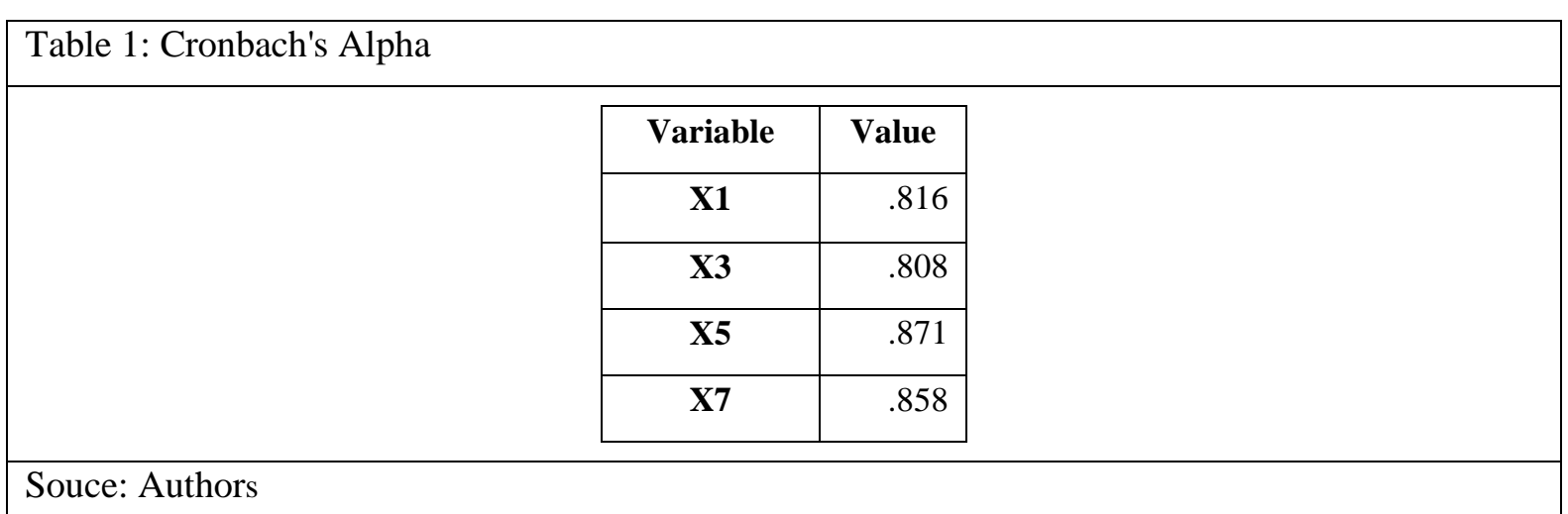

\section{Results of statistical analysis}

This section discusses the results of sequential analyzes and statistical tests described in the research design, and explains the aspects of the methodology applied. The statistical treatment of the data is explained step by step, considering the tests that allow us to accept or reject the research hypothesis

At the end of this section the results are discussed and the validity of the alternative hypothesis is also determined.

\section{Multiple linear regression model}

The technique of multivariate regression was used to define the significance level according to the value of $p=0.05$. For purposes of testing the hypothesis, a multiple regression model for all variables was run. The model includes seven variables according to results of multiple linear regression, two of these were not significant, i.e. ignorance of the location where information (X2) and information security (X7) are stored.

According to the predictors, (Constant) X3, X6, X4, X5, X1, the coefficient of $\mathrm{R}^{2}$ is equal to .632 allowing the independent variables to influence the dependent variable by $60 \%$. Table 2 summarizes the model results.

\begin{tabular}{|c|c|c|}
\hline & R-Squared & .632 \\
\hline & Standard Deviation & 0.89 \\
\hline
\end{tabular}

The value obtained for the statistic F0 190.250 reveals that the model is significant, with five degrees of freedom in the numerator and 553 degrees of freedom in the denominator.

Predictors: (Constant) X3, X6, X4, X5, X1

Dependent variable: $\mathrm{Y}$

The validity of the Betas and the relative importance of the equation thereof was analyzed.

All Betas were significant and, in accordance with their values most important to examine, the extent of use of cloud computing variables are determined in order of importance as follows:

$\mathrm{Y}=\mathrm{X} 3$ (Service Knowledge) $\rightarrow \mathrm{X} 4$ (Legal Knowledge) $\rightarrow \mathrm{X} 5$ (Services Offered by suppliers) $\rightarrow \mathrm{X} 6$ (Cost) $\rightarrow$ X1 (Dependency of Providers) 
CBU I NTERNATIONAL CONFERENCE ON I NNOVATION, TECHNOLOGY TRANSFER AND EDUCATION

\begin{tabular}{|c|r|r|r|r|r|}
\hline \multicolumn{7}{|l|}{ Table 3: Anova } & Sum of squared & \multicolumn{1}{|c|}{ GI } & Mean Square & F & Significance \\
\hline Regression & 767.337 & 5 & 153.467 & 190.250 & .000 \\
\hline Residual & 446.083 & 553 & .807 & & \\
\hline Total & 1213.420 & 558 & & & \\
\hline Source: Authors & & & & \\
\hline
\end{tabular}

Table 4 summarizes the model coefficients.

\begin{tabular}{|c|r|r|r|r|r|r|r|}
\hline \multicolumn{7}{|l|}{ Table 4: Coefficients } \\
\hline Model & Beta & Std. Error & $\begin{array}{c}\text { Standarized } \\
\text { Beta }\end{array}$ & \multicolumn{1}{|c|}{ t } & \multicolumn{1}{c|}{ Sig. } & Tolerance & VIF \\
\hline X3 & .569 & .033 & .491 & 17.053 & .000 & .802 & 1.247 \\
\hline X6 & .220 & .037 & .181 & 5.908 & .000 & .707 & 1.414 \\
\hline X4 & .408 & .035 & .323 & 11.754 & .000 & .879 & 1.138 \\
\hline X5 & .365 & .047 & .255 & 7.771 & .000 & .616 & 1.623 \\
\hline X1 & .128 & .046 & .082 & 2.798 & .005 & .777 & 1.287 \\
\hline Source: Author. & & & & & \\
\hline
\end{tabular}

\section{Conclusion}

This research paper reviews the critical factors that were significant in cloud computing. By applying a linear regression to validate the instrument, it was found that the Cronbach's alpha was satisfactory. It was calculated, after a model was defined, based on the value of $\mathrm{R}^{2}$ (.632).

Five of seven critical factors (variables) were considered for the model based on their significance for the case study of large and medium-sized enterprises in the metropolitan area of Monterrey.

These variables are listed in their order of importance:

- X3 (Knowledge Service)

- X4 (Legal Knowledge)

- X5 (Services Offered by suppliers)

- X6 (Cost)

- X1 (Dependency of Providers)

Cloud computing comprises of a variety of solutions that companies offer in a virtually convenient way, while reducing unnecessary maintenance cost and other costs associated with infrastructure software licenses, hardware, data security, etc. Future suppliers could integrate this technology into creating more cost-effective business models that would meet the fast-paced, dynamic needs of companies. 


\section{References}

Bologna, J., \& Walsh, A. M. (1997). The Accountant's Handbook of Information Technology. New York, United States of America: John Wiley \& Sons, Inc.

Géczy, P., Izumi, N., \& Hasida, K. (2011). Cloudsourcing: Managing Cloud Adoption. Global Journal of Business Research, 6, 57-70.

ISACA (2011). IT Risk/Reward Barometer-Mexico Edition.

Julisch, K., \& Hall, M. (2010). Security and control in the cloud. Information Security. A Global Perspective, 6, 299-309.

Mather, T., Kumaraswamy, S., \& Latif, S. (2009). Security Management in the Cloud. In M. Loukides (Ed.), Cloud Security and Privacy: An Enterprise Perspective on Risks and Compliance (pp. 104-141). California, United States of America: O’Reilly Media, Inc.

Rawal, A. (2011). Adoption of Cloud Computing in India. Journal of Technology Management for Growing Economies, 2, 65-78.

Sheelvant, R. (2009). 10 Things to Know about Cloud Computing Strategy. IT Strategy.

Steven, A. (1999). Information systems: A management perspective. Indiana, United State of America: Addison Wesley.

Suarez, M. (2004). Interaprendizaje Holístico de Matemática. Ibarra, Ecuador: Gráficas Planeta. 